\title{
VARIABILIDADE PLUVIOMÉTRICA E A PRODUÇÃO DE MILHO NO CURIMATAÚ OCIDENTAL DA PARAIÍBA
}

\author{
Maciel Rocha da Silva ${ }^{1}$ \\ Igor Revelles Gomes Luna ${ }^{2}$ \\ João Paulo de Oliveira Santos ${ }^{3}$ \\ Daniel Duarte Pereira ${ }^{4}$ \\ Izaias Romário Soares do Nascimento ${ }^{5}$ \\ Diego Alves Monteiro da Silva ${ }^{6}$ \\ Paulo Henrique de Almeida Cartaxo ${ }^{7}$ \\ Erasmo Venâncio de Luna Neto ${ }^{8}$ \\ Andressa Kamila Souza Alves ${ }^{9}$ \\ José Rayan Eraldo Souza Araújo ${ }^{10}$
}

\section{RESUMO}

O milho é uma das culturas mais cultivadas do Semiárido no Brasil, no entanto, sua produção é ainda pouco tecnificada, o que leva essa cultura ser extremamente dependente de fatores ambientais. Nesse sentido, o presente estudo objetivou analisar a evolução da produção de milho no munícipio de Barra de Santa Rosa, Paraíba, entre os anos de 2002 e 2016, associando os resultados obtidos com a precipitação pluviométrica local. Para tanto, visando analisar a influência da precipitação sobre as variáveis de produção da cultura do milho, assim como a relação entre essas variáveis, utilizou-se uma matriz de correlação. Foi também realizada análise de similaridade entre as variáveis obtidas para os anos avaliados e uma análise descritiva de componentes principais. Os resultados evidenciaram correlação positiva da precipitação pluviométrica sobre todas as variáveis analisadas, principalmente para a área plantada e área colhida. Mesmo em anos com precipitação normal ou acima da média climatológica, a produção total de milho se mostrou baixa. A análise de

\footnotetext{
${ }^{1}$ Engenheiro Agrônomo formado pela Universidade Federal da Paraíba. E-mail: macielr87@gmail.com

${ }^{2}$ Mestrando em Engenharia Ambiental na Universidade Federal Rural de Pernambuco. E-mail: revellesigor@yahoo.com.br

${ }^{3}$ Doutorando em Agronomia na Universidade Federal da Paraíba. E-mail: jpos@agronomo.eng.br 4Professor Associado I do Centro de Ciências Agrárias da Universidade Federal da Paraíba. Email: danielduartepereira@hotmail.com

${ }_{5}^{5}$ Mestrando em Agronomia na Universidade Federal da Paraíba. E-mail: izaias.agronomia@gmail.com diegoalves1903@gmail.com

${ }^{7}$ Mestrando em Agronomia na Universidade Federal da Paraíba. E-mail:

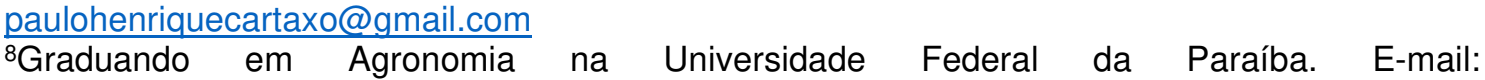
erasnetinhow@gmail.com

${ }^{9}$ Doutoranda em Genética e Melhoramento na Universidade Federal de Viçosa. E-mail: andressak.agro@gmail.com

${ }^{10}$ Graduando em Agronomia na Universidade Federal da Paraíba. E-mail: rayanccaufpb@gmail.com 
componentes principais registrou $84,67 \%$ de explicação da variância original nos dois primeiros eixos e demonstrou a forte associação entre área plantada, colhida e produção. A análise de agrupamento hierárquico gerou a formação de quatro grupos principais, sendo estes associados principalmente aos resultados produtivos da cultura do milho. A precipitação pluviométrica se mostrou como um fator extremamente importante na dinâmica de produção de milho no município de Barra de Santa Rosa, influenciando principalmente na área plantada e colhida.

Palavras-chave: Agricultura familiar; Mudanças climáticas; Zea mays L. 


\title{
PLUVIOMETRIC VARIABILITY AND CORN PRODUCTION IN THE WESTERN CURIMATAÚ OF PARAIIBA
}

\begin{abstract}
Corn is one of the most cultivated crops of the semiarid region in Brazil, however, its production is still poorly technified, which makes it extremely dependent on environmental factors. In this sense, the present study aimed to analyze the evolution of corn production in the municipality of Barra de Santa Rosa, Paraíba, between 2002 and 2016, associating the results obtained with local rainfall. In order to analyze the influence of precipitation on the variables of corn crop production, as well as the relationship between these variables, a correlation matrix was used. Similarity analysis was also performed between the variables obtained for the years evaluated and a descriptive analysis of principal components. The results showed a positive correlation of rainfall over all variables analyzed, especially for the planted area and harvested area. Even in years with normal rainfall or above the climatological average, total maize production was low. Principal component analysis recorded $84.67 \%$ explanation of the original variance in the first two axes and showed the strong association between planted area, harvested area and yield. The hierarchical cluster analysis generated the formation of four main groups, which were mainly associated with the productive results of corn crop. Rainfall was an extremely important factor in the dynamics of corn production in the municipality of Barra de Santa Rosa, mainly influencing the planted and harvested area.
\end{abstract}

Keywords: Family farming; Climate changes; Zea mays L.

\section{INTRODUÇÃO}

O Semiárido do Brasil (SAB) abrange 1.262 municípios nos estados de Alagoas, Bahia, Ceará, Maranhão, Minas Gerais, Paraíba, Pernambuco, Piauí, Rio Grande do Norte e Sergipe, é nessa região que se concentra a maior população rural do Brasil (MARINHO et al., 2019). No SAB a água é um fator limitante para o abastecimento humano e dessedentação animal, assim como para as atividades de produção agropecuária, atividades estas que são pautadas em sistemas de exploração arcaicos e pouco tecnificados, tornando a agricultura local uma atividade de risco e altamente susceptível a eventos de estiagem (BRITO et al., 2012).

Durante o período de 1991 a 2012, os registros oficiais apontaram 19.517 ocorrências de eventos de seca no Brasil, que em sua grande parte se concentraram no Nordeste do país. No SAB, a seca que teve início em 2011, intensificou-se em 2012 e continuou até 2017, sendo considerada a mais severa 
das últimas décadas, gerando impactos na maioria dos municípios dessa região e afetando diretamente quase 9 milhões de pessoas (BRITO et al., 2017). Esses eventos, em média, são os riscos naturais mais caros do Brasil, principalmente por seus reflexos na produção agrícola e animal, apenas a seca de 2012/2013, por exemplo, resultou em perdas econômicas de US \$1,6 bilhão para as 10 culturas mais importantes do país (feijão, arroz, milho, algodão, banana, canade-açúcar, mandioca, soja e café), US \$1,5 bilhão devido à mortalidade do gado e custos superiores a US \$1,5 bilhão em sinistros de seguros (CUNHA et al., 2015).

Além dos efeitos diretos, em períodos de extrema seca, a segurança alimentar das comunidades rurais do Semiárido torna-se ainda mais vulnerável, principalmente devido à redução de renda e aumento de preços dos produtos agrícolas. A inflação dos preços dos alimentos, associada à perda de receita devido à queda na colheita e perda de produtividade, resulta em diversos problemas de ordem social, como a fome e êxodo rural (ALVALA et al., 2017).

O milho (Zea mays L.) é uma das culturas mais importantes para o Semiárido do Brasil, fazendo parte da alimentação básica da população local e sendo um dos principais constituintes para a alimentação animal, no entanto, embora essa cultura seja tolerante a uma ampla faixa de temperaturas, é sensível ao estresse hídrico do solo (MARTINS et al., 2018). Em geral, para o milho atingir o seu rendimento máximo, requer valores de precipitação pluviométrica entre 500 a $800 \mathrm{~mm}$, sendo necessário que essa chuva seja bem distribuída dentro de suas fases fenológicas; eventos de estiagem prolongadas são especialmente nocivos a essa cultura durante os períodos de floração e de formação dos grãos (ROSSATO et al., 2017).

Estudos como o de Dias e Silva (2015), evidenciam a vulnerabilidade da produção de milho no Semiárido à ocorrência de precipitações pluviométricas satisfatórias, mostrando que essa variável é determinante para o dimensionamento da área plantada e nos índices produtivos. Nesse sentido, tomando como base a importância da cultura do milho para o SAB, os estudos dos impactos dos eventos de seca sobre essa cultura são necessários para a delimitação de estratégias de produção mais eficientes e adaptadas as 
condições locais, como por exemplo, através da seleção de variedades mais tolerantes ao déficit hídrico e da escolha das melhores técnicas a serem adotadas. Assim, o presente trabalho objetivou avaliar a evolução da produção de milho (Zea mays L.) durante o período de doze anos no munícipio de Barra de Santa Rosa, Paraíba, associando-se os resultados obtidos com os padrões anuais de precipitação pluviométrica local.

\section{MATERIAL E MÉTODOS}

O município de Barra de Santa Rosa (Figura 1) está localizado na microrregião do Curimataú Ocidental da Paraíba. O município possui uma área de 825,01 km² e está localizado entre as coordenadas 643'12" S e 36³'39" W, com uma altitude de aproximadamente $457 \mathrm{~m}$ e inserido nos domínios da bacia hidrográfica do rio Curimataú (IBGE, 2018). Conforme a classificação de Köppen, o tipo climático da região é Bsh, semiárido quente, com chuvas de verão/outono, e precipitação média em torno de $400 \mathrm{~mm}$ anuais (FURTADO et al., 2011).

Figura 1 - Localização do município de Barra de Santa Rosa, Paraíba.
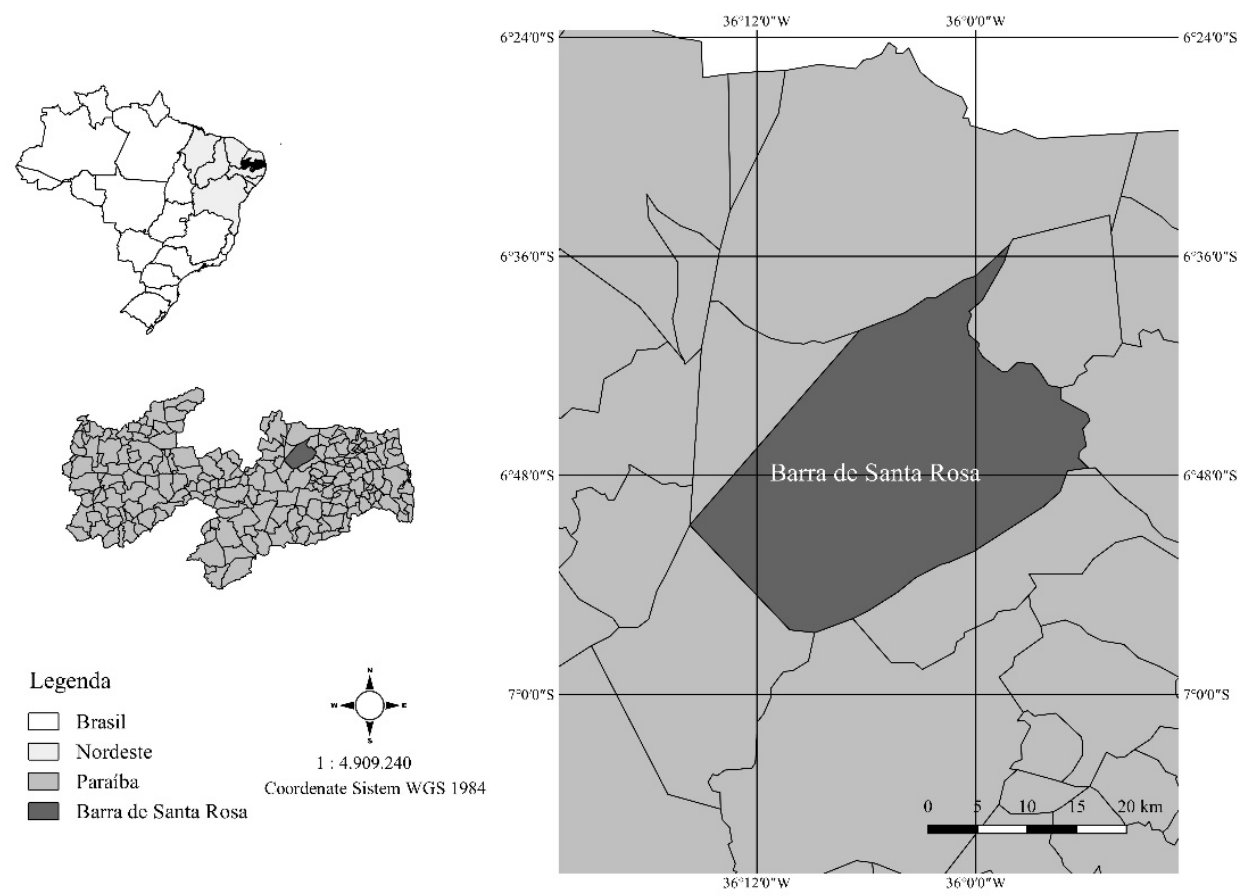
As informações referentes ao cultivo de milho em Barra de Santa Rosa, no período de 2005 a 2016, foram obtidas através do banco de dados da Produção Agrícola Municipal - Lavoura Temporária do IBGE, sendo para isso utilizado o Sistema de Recuperação Automática (SIDRA). Os valores relativos à precipitação pluviométrica no período estudado, foram obtidos junto ao site da Agência Executiva de Gestão das Águas do Estado da Paraíba (AESA).

Para analisar a influência da precipitação sobre as variáveis de produção da cultura do milho, assim como a relação entre essas variáveis, foi utilizada uma matriz de correlação. Foi também realizada análise de similaridade entre as variáveis obtidas para os anos avaliados e uma análise descritiva de componentes principais (ACP). O programa utilizado foi o $\mathrm{R}$ ( $\mathrm{R}$ Development CoreTeam, 2009).

\section{RESULTADOS E DISCUSSÃO}

A precipitação pluviométrica no período em estudo variou de $824,9 \mathrm{~mm}$ em 2004 a 156,8 mm em 2012 (Tabela 1). Dos 15 anos em monitoramento, 10 deles (2003, 2005, 2006, 2007, 2010, 2012, 2013, 2014, 2015 e 2016) apresentaram acumulados anuais de chuvas inferiores à média climatológica local, que é de 364,9 mm (AESA, 2019). A ocorrência regular de chuvas é considerada a variável meteorológica mais importante para as atividades agrícolas, abastecimento de água e vida humana; dessa forma, a compreensão do comportamento espaço-temporal dessa variável é essencial para a identificação da ocorrência de fenômenos extremos, como as secas (SANTOS et al., 2019).

De modo geral, o clima da região Semiárida é caracterizado por um curto período chuvoso e esse período está sujeito a elevada variabilidade interanual; assim, desde o período da colonização, regularmente secas severas são responsáveis por causarem prejuízos as atividades agropecuárias locais (PILZ et al., 2019), e influenciarem a dinâmica produtiva dessa área. Registrou-se fortes oscilações na área plantada com milho no município de Barra de Santa Rosa, com máximos de 1500 hectares anuais e mínimos de 300 hectares (Tabela 1). 


\section{১unisul}

Tabela 1 - Precipitação pluviométrica e variáveis de produção de milho no município de Barra de Santa Rosa - PB, durante o período de 2002 a 2016.

\begin{tabular}{lccccc}
\hline Ano & $\begin{array}{c}\text { Precipitaçã } \\
\text { o (mm) }\end{array}$ & $\begin{array}{c}\text { A. } \\
\text { Plantada } \\
\text { (ha) }\end{array}$ & $\begin{array}{c}\text { A. Colhida } \\
\text { (ha) }\end{array}$ & $\begin{array}{c}\text { Produçã } \\
\mathbf{o}(\mathbf{t})\end{array}$ & $\begin{array}{c}\text { Produtividad } \\
\text { e (kg.ha-1) }\end{array}$ \\
\hline $\mathbf{2 0 0 2}$ & 416.4 & 1300 & 100 & 20 & 100 \\
$\mathbf{2 0 0 3}$ & 346.1 & 1300 & 1300 & 390 & 200 \\
$\mathbf{2 0 0 4}$ & 824.9 & 1500 & 1500 & 300 & 250 \\
$\mathbf{2 0 0 5}$ & 349.4 & 1500 & 1500 & 600 & 600 \\
$\mathbf{2 0 0 6}$ & 227 & 1500 & 1500 & 900 & 200 \\
$\mathbf{2 0 0 7}$ & 351.2 & 1100 & 1100 & 880 & 400 \\
$\mathbf{2 0 0 8}$ & 626.3 & 1500 & 1500 & 1200 & 300 \\
$\mathbf{2 0 0 9}$ & 647.7 & 1500 & 1300 & 620 & 300 \\
$\mathbf{2 0 1 0}$ & 321.7 & 800 & 400 & 96 & 100 \\
$\mathbf{2 0 1 1}$ & 683.1 & 1300 & 1300 & 540 & 300 \\
$\mathbf{2 0 1 2}$ & 156.8 & 300 & 100 & 10 & 50 \\
$\mathbf{2 0 1 3}$ & 219.8 & 800 & 800 & 480 & 400 \\
$\mathbf{2 0 1 4}$ & 173.5 & 900 & 900 & 360 & 200 \\
$\mathbf{2 0 1 5}$ & 203.2 & 320 & 320 & 29 & 50 \\
$\mathbf{2 0 1 6}$ & 207.3 & 750 & 750 & 113 & 220 \\
\hline Fonte: SIDRA-IBGE; AESA. & & & & \\
\hline
\end{tabular}

A área colhida apresentou comportamento semelhante a área plantada, porém, quatro anos (2002, 2009, 2010 e 2012), apresentaram discrepâncias com essa primeira variável. No ano de 2012, por exemplo, além da forte redução na 
área plantada quando comparada ao ano anterior, a área colhida foi ainda menor, o que está fortemente relacionado aos baixos índices pluviométricos observados para esse ano em questão. Eventos de instabilidade climática, principalmente a ocorrência concentrada de chuvas em determinados períodos em detrimento de outros dentro do ciclo produtivo do milho, como por exemplo durante o plantio, levam ao plantio de áreas maiores, porém, devido ao déficit hídrico que a cultura é submetida posteriormente, as áreas colhidas tendem a serem reduzidas, sendo as perdas econômicas ainda maiores em anos de secas recorrentes (SENA et al., 2019). Resultados semelhantes foram observados por Batista et al. (2018) para a produção de feijão no município de Itaporanga, Semiárido da Paraíba.

A produção municipal de milho foi relativamente pequena, como valores máximos de 1200 toneladas. Boa parte dos anos apresentou produção total abaixo de 500 toneladas, com destaque para o período de 2012 a 2016, em que a produção se manteve constantemente baixa.

Mesmo em períodos em que a precipitação pluviométrica foi superior à média climatológica anual, a produtividade do milho em Barra de Santa Rosa foi inferior à média nacional (5104 kg.ha-1 $)$, do Nordeste (2593 kg.ha-1) e do estado da Paraíba (600 kg.ha-1) (SIDRA, 2018). Resultados que atestam a baixa tecnificação empregada para essa cultura, o que a torna ainda mais susceptível a eventos de natureza climática, como por exemplo, estiagens prolongadas. Embora o milho seja tolerante a uma ampla faixa de temperaturas, é sensível a eventos de déficit hídrico do solo, fator que leva essa cultura a apresentar grandes oscilações interanuais em sua produção na região Semiárida do Brasil (MARTINS et al., 2018).

A matriz de correlação entre as variáveis de produção e precipitação (Figura 2), evidencia uma correlação significativa e positiva entre a precipitação e área plantada $(r=0,68)$, assim como com a área colhida $(r=0,54)$, demonstrando a necessidade de bons índices pluviométricos para que os agricultores locais ampliem suas áreas de produção e as plantas de milho possam ter seu pleno desenvolvimento e cheguem ao ponto de colheita. Todavia, a correlação entre precipitação e produção, e precipitação e 
produtividade é fraca, o que se deve ao modelo arcaico ainda empregado pelos agricultores locais, em que outros agentes, como por exemplo a falta de insumos, é responsável por baixos índices produtivos (BATISTA et al., 2018).

Figura 2 - Matriz de Correlação entre as Variáveis Analisadas.

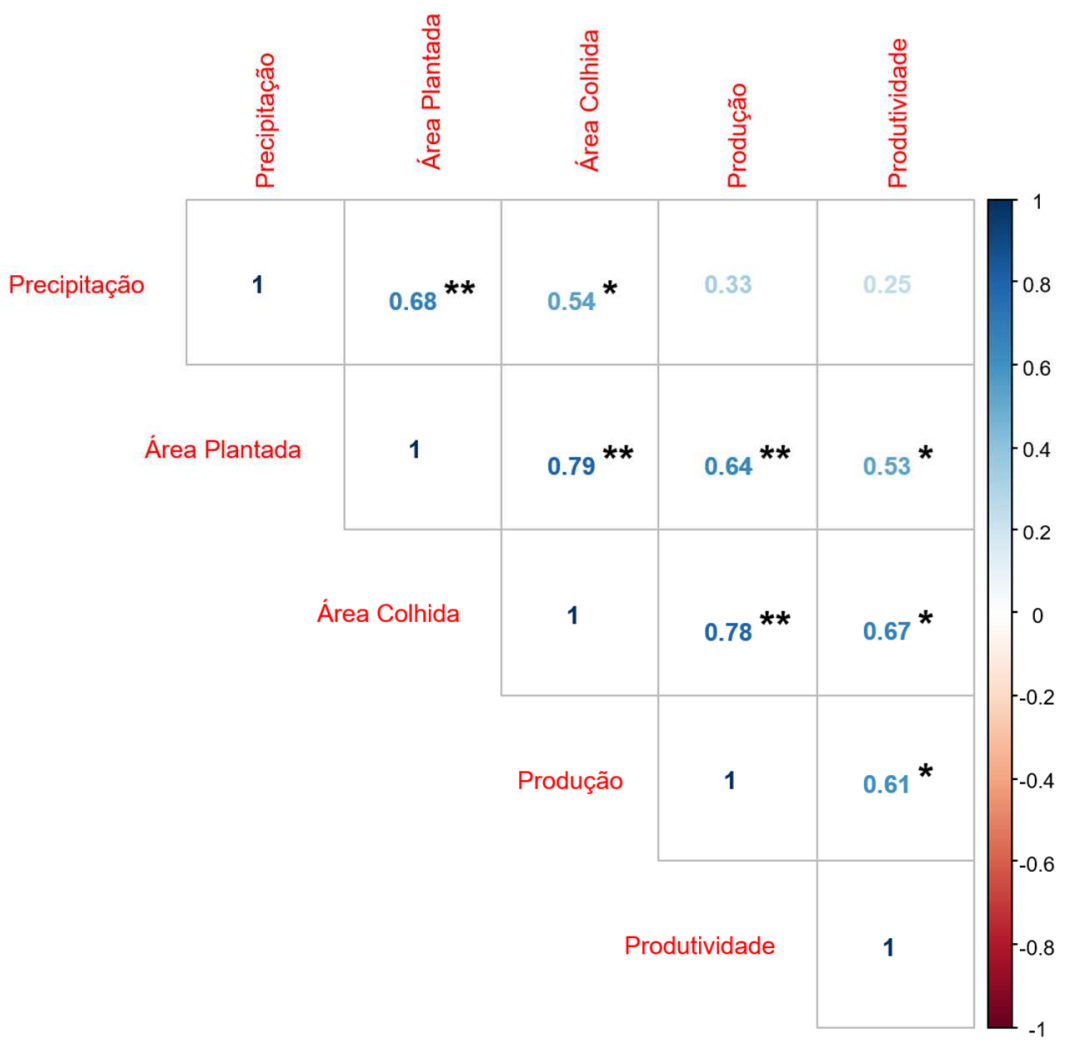

$* ; *$ : Significativo a 5 e $1 \%$, respectivamente.

A área plantada apresentou correlação significativa $(p<0,01)$ e forte com a área colhida $(r=0,79)$, assim como a área colhida com a produção $(r=0,78)$, o que já era esperado, visto essas variáveis apresentarem um grau de dependência. Nas condições produtivas do Semiárido brasileiro, a ocorrência de perdas totais ou parciais da área plantada, refletindo assim na área colhida e na produção final, estão associada a diversos eventos, merecendo destaque os eventos climatológicos e interferência de alta intensidade de pragas e doenças (DIAS; SILVA, 2015).

A análise de componentes principais (Figura 3) registrou $84,67 \%$ de explicação da variância original nos dois primeiros eixos. Para o eixo 1 , que 
explicou 67,43\% da variância, as variáveis com maior associação foram a área colhida $(r=-0,50)$, área plantada $(r=-0,48)$ e produção $(r=-0,45)$, comportamento também observado por Farias et al. (2017) para a produção de milho em Boqueirão no estado na Paraíba. Nesse eixo, registrou-se o agrupamento dos anos de 2012 e 2015, cuja área colhida, plantada e produção foram as menores de todo o período amostral.

Figura 3 - Análise de Componentes Principais (ACP). AC = Área Colhida; AP = Área Plantada; Precip. = Precipitação Pluviométrica; Produção = Quantidade Produzida; Produtiv. $=$ Produtividade Média.

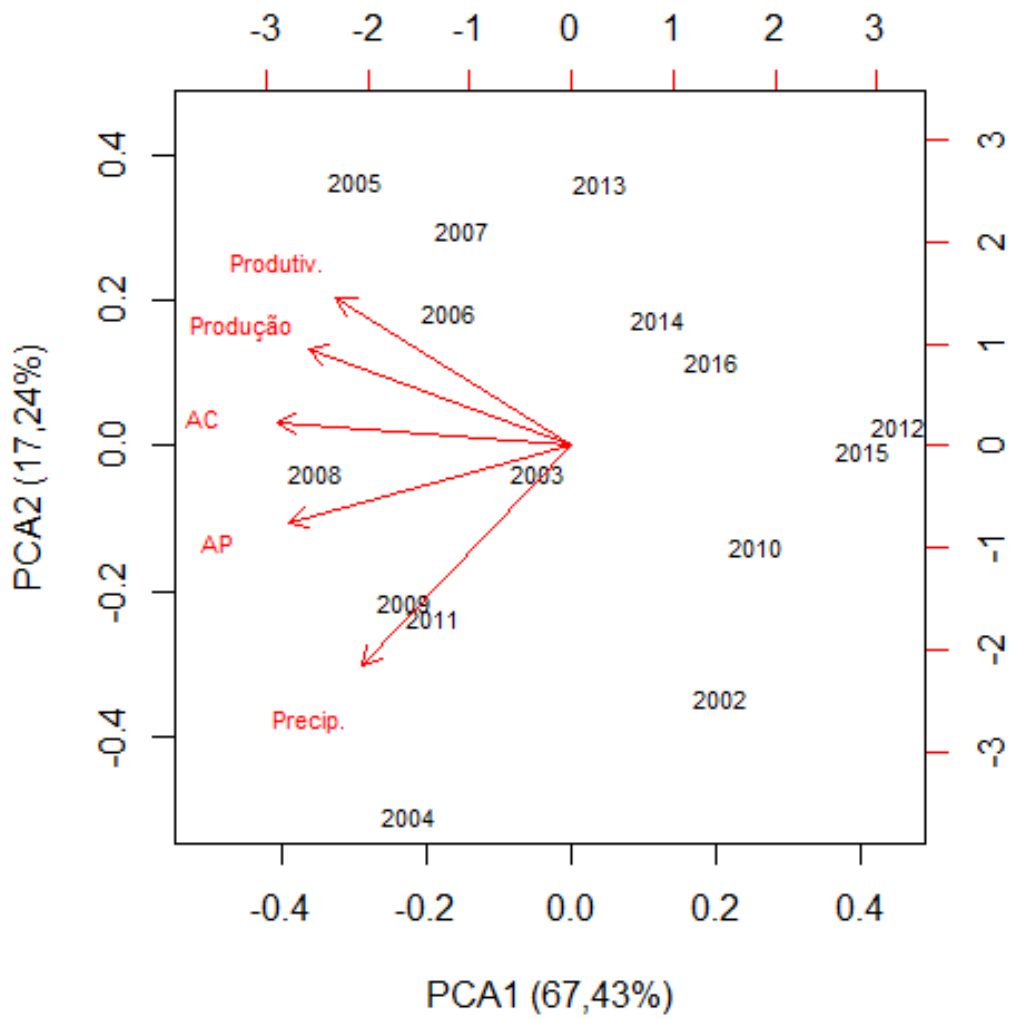

Para o eixo 2, com 17,24\% de explicação, observa-se a influência negativa da precipitação $(r=-0,74)$ sobre a produtividade $(r=0,50)$. Observou-se a formação de um agrupamento dos anos de 2005, 2007 e 2013, anos esses que apresentaram as maiores médias de produtividade, no entanto, apresentaram 


\section{GESTÃO \& SUSTENTABILIDADE} AMBIENTAL

\section{sunisul}

valores de precipitação pluviométrica inferiores a outros anos e a média climatológica local.

A análise de agrupamento hierárquico (Figura 4), considerando no dendograma um ponto de quebra na distância de 800 , gerou a formação de quatro grupos principais, sendo estes associados principalmente aos resultados produtivos da cultura do milho. Merece destaque o grupo formado pelo ano de 2002, que embora tenha apresentado regime de chuvas dentro da média climatológica local, exibiu baixa produção total e baixa produtividade, tal resultado se explica pelo fato que essa chuva pode ter se concentrado em um curto período de tempo durante o ciclo dessa cultura, o que pode levar a problemas no desenvolvimento vegetativo e produtivo dessas plantas (PEREIRA et al., 2014).

Figura 4 - Análise de Agrupamento Hierárquico entre os anos monitorados quanto as variáveis analisadas.

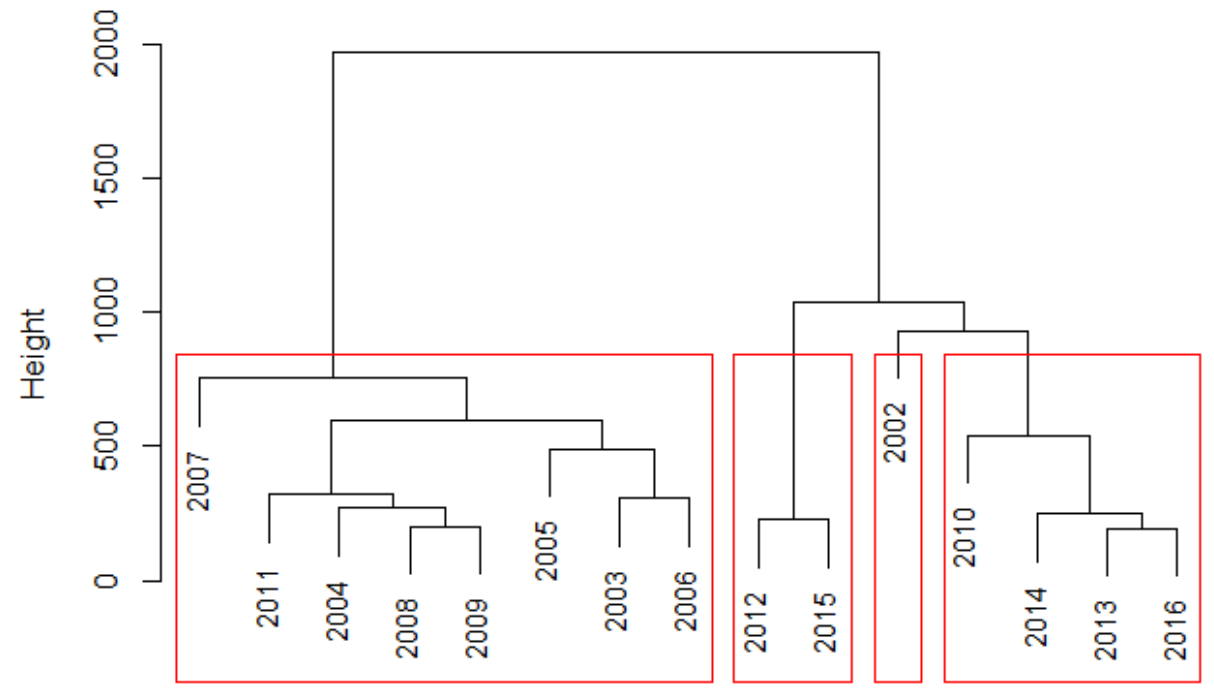

Matriz de similaridade

A geração de grupos como o formado para os anos de 2012 e 2015, evidencia a elevada vulnerabilidade produtiva que a cultura do milho enfrenta na região Semiárida. Análises que buscam avaliar as perdas médias das culturas 
temporárias em municípios do Semiárido em função de eventos de estiagens prolongadas, apontam o milho como umas das lavouras mais vulneráveis, com 80,3\% desses municípios com perdas nessa cultura em anos secos (LIMA et al., 2016).

\section{CONSIDERAÇÕES FINAIS}

A precipitação pluviométrica se mostrou como um fator extremamente importante na dinâmica de produção de milho no município de Barra de Santa Rosa, influenciando principalmente na área plantada e colhida. Estratégias de produção menos dependentes das condições climáticas precisam ser implantadas no município em questão visando reduzir a vulnerabilidade produtiva local dessa cultura.

\section{REFERÊNCIAS}

AESA. Agência Executiva de Gestão das Águas do Estado da Paraíba. Monitoramento. Disponível em: http://www.aesa.pb.gov.br/. Acesso em 12 de outubro de 2019.

ALVALA, R.; CUNHA, A. P.; BRITO, S. S.; SELUCHI, M. E.; MARENGO, J. A.; MORAES, O. L.; CARVALHO, M. A. Drought monitoring in the Brazilian Semiarid region. Anais da Academia Brasileira de Ciências, v. 91, n.1, e20170209, 2017.

BATISTA, M. C.; SANTOS, J. P. O.; SILVA FILHO, J. A.; SOUSA, J. I.; FELIX, R. J. S.; SILVA, J. L. C. Influence of rainfall variability on bean production (Phaseolus vulgaris L.) in a municipality of Brazilian semiarid. Journal of Environmental Analysis and Progress, v. 3, n. 1, p. 001-007, 2018.

BRITO, L. T. L.; CAVALCANTI, N. B.; SILVA, A. S.; PEREIRA, L. A. Produtividade da água de chuva em culturas de subsistência no Semiárido Pernambucano. Engenharia Agrícola, v. 32, n. 1, p. 102-109, 2012.

BRITO, S. S. B.; CUNHA, A. P. M.; CUNNINGHAM, C. C.; ALVALÁ, R. C.; MARENGO, J. A.; CARVALHO, M. A. Frequency, duration and severity of drought in the Semiarid Northeast Brazil region. International Journal of Climatology, v. 38, n. 2, p. 517-529, 2018.

CUNHA, A. P. M.; ALVALÁ, R. C.; NOBRE, C. A.; CARVALHO, M. A. Monitoring vegetative drought dynamics in the Brazilian semiarid region. Agricultural and Forest Meteorology, v. 214, p. 494-505, 2015.

DIAS, R. S.; SILVA, D. F. Relação entre variabilidade pluviométrica, indicadores socioeconômicos e produção agrícola no Cariri/Centro Sul cearense. Ambiência, v.1, n.2, p. 345-358, 2015. 
FARIAS, A. A.; SOUSA, F. A. S.; MORAES NETO, J. M.; ALVES, A. S. Secas e seus impactos no município de Boqueirão, PB, Brasil. Ambiente \& Água, v.12, n.2, p.316- 330, 2017.

FURTADO, D. A.; CARVALHO JUNIOR, S. B.; LIMA, I. S. P.; COSTA, F. G. P.; SOUZA, J. G. Desempenho de frangos alimentados com feno de maniçoba no semiárido paraibano. Revista Brasileira de Ciências Agrárias, v. 6, n. 4, p. 722-728, 2011.

IBGE. Instituto Brasileiro de Geografia e Estatística. Infográficos. 2018. Disponível

em: https://cidades.ibge.gov.br/brasil/pb/barradesantarosa/panorama. Acesso em 21 de setembro de 2019.

LIMA, P. V. P. S.; MENDES, C. M.; ROCHA, L. A.; OLIVEIRA, M. R. R. No Rastro da Vulnerabilidade às Secas: Uma Análise da Produção de Grãos no Semiárido Brasileiro. REDM, v. 19, n. 1, p.183-196, 2016.

MARINHO, J. O.; CAMPOS, J. O; LIMA, V. R. P. A importância das cisternas de placas na zona rural de Serra Redonda-PB: uma análise da comunidade Torre. Revista Geotemas, v. 9, n. 1, p. 7-27, 2019.

MARTINS, M. A.; TOMASELLA, J.; RODRIGUEZ, D. A.; ALVALÁ, R. C.; GIAROLLA, A.; GAROFOLO, L. L. et al. Improving drought management in the Brazilian semiarid through crop forecasting. Agricultural Systems, v. 160, p. 2130, 2018.

PEREIRA, V. G. C.; GRIS, D. J.; MARANGONI, T.; FRIGO, J. P.; AZEVEDO, K. D.; GRZESIUCK, A. E. Exigências agroclimáticas para a cultura do feijão (Phaseolus vulgaris L.). Revista Brasileira de Energias Renováveis, v. 3, n. 1, p. 32-42, 2014.

PILZ, T.; DELGADO, J. M.; VOSS, S.; VORMOOR, K.; FRANCKE, T.; COSTA, A. C. et al. Seasonal drought prediction for semiarid northeast Brazil. Hydrology and Earth System Sciences, v. 23, p. 1951-197, 2019.

R DEVELOPMENT CORE TEAM.R Foundation For Statistical Computing. R: A Language and Environment for Statistical Computing. Viena, Áustria. 2009. ISBN: 3-900051-07-0. Disponível em: http://www.R-project.org. Acesso em 12 de outubro de 2019.

ROSSATO, L.; ALVAlÁ, R. C.; MARENGO, J. A.; ZERI, M.; CUNHA, A. P.; PIRES, L.; BARBOSA, H. A. Impact of soil moisture on crop yields over Brazilian semiarid. Frontiers in Environmental Science, v. 5, n. 73, p. 1-16, 2017.

SANTOS, C. A. G.; MOURA, R.; SILVA, R. M.; COSTA, S. G. F. Cluster Analysis Applied to Spatiotemporal Variability of Monthly Precipitation over Paraíba State Using Tropical Rainfall Measuring Mission (TRMM) Data. Remote Sensing, v. 11, n. 6, e637, 2019. 


\section{¿unisul}

SENA, J. P. O.; MORAES NETO, J. M.; LUCENA, D. B. Variabilidade da Precipitação em Sumé e São João do Cariri e suas Consequências na Agropecuária. Revista Brasileira de Climatologia, v. 25, p. 278-293, 2019.

SIDRA - Sistema IBGE de Recuperação Automática. Produção Agrícola Municipal. 2018. Disponível em: https://sidra.ibge.gov.br/pesquisa/pam/tabelas. Acesso em 12 de outubro de 2019. 